\title{
ON THE STABILITY OF CIRCUIT-SWITCHED NETWORKS WITH NON-HIERARCHICAL ROUTING
}

\author{
Lorne G. Mason
}

INRS-Telecommunications

3 Place du Commerce

Verdun, Quebec H3E $1 \mathrm{H} 6$

\section{Summary}

The stability of non-hierarchical circuit-switched networks is considered. New results are derived, using the equilibrium flow technique, which relate various parameters to network stability. It is demonstrated that instability is a pervasive phenomenon in networks with mutual overflow, while trunk reservation and route cancellation provide effective means to stabilize an otherwise unstable network. A simple expression is derived for a stabilizing reservation level.

\section{Introduction}

Instabilities in networks, employing non-hierarchical routing. were first reported by Nakagomi and Mori ${ }^{1}$. Krupp ${ }^{2}$, presented simulation results which exhibited bistable behavior in networks with non-hierarchical routing. He also demonstrated that a small amount of trunk reservation removed this undesirable feature. Marbukh ${ }^{3}$ carried out a dynamic analysis of the asymptotic network, which contains an infinite number of nodes and bypass routes. In ${ }^{4}$ an equilibrium flow analysis was made for uniform symmetric networks with a finite number of alternate routes. Akinpelu ${ }^{5}$, has considered the performance of engineered networks with non-hierarchical routing and trunk reservation.

Several characterizations of network instability are possible 6 . The list includes : non-uniqueness of the equilibrium flow pattern; multi-modality in the state probability distribution eigenvalues corresponding to dynamic modes with long time constants; bifurcation in the dynamic state phase trajectory; discontinuities in the input-state-throughput characteristics; hysteresis in the static load-loss or load-state relation.

Several techniques have been employed to investigate the stability of multi-access networks ${ }^{6}$, including exact and approximate static models. transient analyses, and catastrophe theory; where it has been shown that the equilibrium flow technique provides reasonably accurate predictions in regard to network stability. In the context of non-hierarchical circuit-switched networks, all of the analyses so far reported, employ approximate static or dynamic models due to the large state space associated with an exact analysis.

\section{Static Equilibrium Flow Analysis}

Three different models have been considered to determine the effect of various system parameters on stability. Specifically, the models considered are the 1) uniform symmetric asymptotic network with trunk reservation, 2) uniform symmetric network, with a finite number of alternate routes and 3) small non-uniform networks. The first model serves to confirm that the static equilibrium fow is equivalent to the steady state given by the dynamic analysis reported in ${ }^{3}$. It provides information on the effect of trunk reservation, group size, and path length on system stability. The second model serves to show the effect of alternate route multiplicity (or flow control via route cancellation) and traffic peakedness on network stability. The last model illustrates the effect of non-uniformity in trunk group size and traffic distribution on system stability.

\subsection{The Asymptotic Network Analysis}

Here we consider a complete network consisting of $N \rightarrow \infty$ nodes, where each pair of nodes is connected with a two-way trunk group of $L$ trunks capacity. The exogenous two-way traffic demand between each $O-D$ pair is $\Lambda$ Erlangs. For each trunk group of size $L$, there exists $L-r$ trunks available only to direct traffic. The remaining $r$ trunks are available to both direct and alternate routed traffic. For each alternate route, there are $S$ trunk groups in tandem connecting the considered $O-D$ pair. Exogenous traffic is first offered to the direct group. If it is full, then the call is randomly routed to the set of alternate paths where all links on such paths have an occupancy $X$, less than the reservation threshold, $r$. This traffic distribution can also be interpreted as that obtained by scanning S-hop paths sequentially and routing on the first path were all link occupancies $X<r$. In this latter interpretation we need symmetrical routing to assure all links receive the same offered traffic. Assuming link independence, and Poisson traffics offered to each link, the state dependent birth and death rates are easily obtained by using the symmetry and uniformity. It is straightforward to show that the equilibrium flows satisfy the polynomial

$$
\left(\sum_{i=0}^{r-1} Z^{i} / i !\right)(Z / \Lambda-1)=S Z^{r} \frac{\Lambda^{L-r}}{L !}
$$

where $Z$ is the link offered traffic, in Erlangs, which includes both direct and overflow traffic. Figure 1. shows the relationship between the link offered traffic, $Z$. and the exogenous traffic, $\Lambda$, for various levels of trunk reservation, $r$

\section{Stabilizing Reservation Threshold}

An analytic approach to determining the system stability is possible, by considering the flow equation (1), as a polynomial in $Z$, given $\Lambda$ as the parameter. Instability now corresponds to a multiple positive roots, $Z(\Lambda)$, for some positive $\Lambda$.

Expanding (1) in descending powers of $Z$ results in

$$
\begin{aligned}
& \left(\frac{1}{(r-1) ! \Lambda}-\frac{S \Lambda^{L-r}}{L !}\right) Z^{r}+\left(\frac{1}{(r-2) ! \Lambda}-\frac{1}{(r-1) !}\right) Z^{r-1} \\
& +\left(\frac{1}{(r-3) ! \Lambda}-\frac{1}{(r-2) !}\right) Z^{r-2} \cdots+\left(\frac{1}{\Lambda}-1\right) Z-1=0
\end{aligned}
$$

We observe that the coefficient of $Z^{k}, k<r$, is positive for $\Lambda<k$ and negative for $\Lambda>k$. Defining a critical traffic level $\Lambda^{*}$ by

$$
\left(\frac{L !}{S(r-1) !}\right)^{\frac{1}{L-r+1}} \triangleq \Lambda^{*}(r)
$$


If $\Lambda>\Lambda^{*}$ the coefficient of $Z^{\prime}$ is negative while if $\Lambda<\Lambda^{*}$ it is positive.

An optimal. reservation threshold, $R^{*}$, exists among the set of stable reservation thresholds. $r^{*}$. where

$$
\begin{aligned}
R^{*} & =\max \left\{r^{*}\right\} \\
r^{*} & =\left\{r \mid \Lambda^{*}(r)-r>-1\right\}
\end{aligned}
$$

For an optimal reservation, where $L-R^{*}$ trunks are reserved for direct traffic only, and all $\Lambda<R^{*}-1$, there is a single sign change in the coefficients implying at most one positive real root. For $\Lambda$ in excess of $R^{*}-1$, there are no positive real solutions. This implies that the throughput is maximized for the reservation level $R^{*}$ as defined by equation (4). This is also the point at which the system becomes stable. If $r>R^{*}$ then two sign changes occur in the coefficients for $\Lambda^{*}(r)<\Lambda<\Lambda^{*}\left(R^{*}\right)$ allowing for multiple positive real roots and instability.

\subsection{Uniform Networks with a Finite Number of}

\section{Alternate Routes}

The underlying network may have a firite or an infinite number of nodes, provided that all links have equal capacity, the networks are complete and two-link sequential routing is employed. The asymptotic network, considered in section 2.1, corresponds to the limiting case of networks considered in this section when the number of alternate routes increases without bound and $S=2$. All results given in this section correspond to the case without trunk reservation. As will be subsequently shown, unstable networks can be stabilized by cancelling a sufficient number of alternate routes. This is a form of flow control which has long been employed in the public switched network.

An analysis was previously made ${ }^{4}$ for the stability of such networks, where the effect of group size, number of alternate routes, and call control procedure (sequential office control, SOC, and originating office control, OOC), on network stability was considered. Here we summarize those results along with the effect of traffic peakedness on system stability.

Figure 2. indicates that increasing trunk group siz $T$, and number of alternate routes, $k$, reduces the stability margin for the OOC call control method. SOC networks are much more stable than OOC networks, which is not surprising as the pocketed calls act as a form of flow control. Peakedness in the traffic streams tends to reduce the stability margin relative to random traffic.

\subsection{The Non-uniform Network}

In order to assess the effect of non-uniformity in trunk group size, $N_{i}$, and traffic demand, $\lambda_{i}$, on stability, a simple asymmetric network was analyzed. Here, the first offered traffic, $\lambda_{i}$, offered to path, $i$. $i=1,2$, overflows to the other group, with the volume of overflow being determined by the Erlang $B$ formula. No trunk reservation was included in the model. The overflow streams are multiplied by the gain factors, $g_{i}$, which can be interpretted as the ratio of the number of hops in the secondary to the primary paths. Alternate routed traffic, which is blocked, is cleared from the system. To assess the stability of the system we derive an expression for the link offered traffic to path 1 , namely, $A_{1}$, in terms of the traffic demand vector, $\lambda_{1}$, and $\lambda_{2}$. This results in a polynomial for $A_{1}$ with $\lambda_{1}$. and $\lambda_{2}$, as parameters in the coefficients. Multiple positive real roots of this polynomial indicate that the system is unstable. Making the appropriate substitutions and rearrangements we obtain a polynomial in $A_{1}$. where we have used the Binomial expansion formula.

$\left(A_{1}-\lambda_{1}\right) \sum_{i=0}^{N_{2}} \lambda_{2}^{i} / i ! \sum_{k=0}^{i}\left(\begin{array}{l}i \\ k\end{array}\right)\left(g_{2} \lambda_{1} A_{1}^{N_{1}} /\left(N_{1} ! \lambda_{2}\right)\right)^{k}\left(\sum_{i=0}^{N_{1}} A_{1}^{i} / i !\right)^{N_{2}-k}$

$$
\begin{gathered}
-g_{1} \lambda_{2}^{N_{2}+1} / N_{2} ! \sum_{i=0}^{N_{2}}\left(\begin{array}{c}
N_{2} \\
k
\end{array}\right)\left(g_{2} \lambda_{1} A_{1}^{N_{1}} /\left(N_{1} ! \lambda_{2}\right)\right)^{k}\left(\sum_{i=0}^{N_{1}} A_{1}^{i} / i !\right)^{N_{2}-k} \\
=0
\end{gathered}
$$

Figure 3. shows the regions of multiple roots for the uniform and nonuniform networks. In the language of catastrophe theory ${ }^{7}$, these regions are the bifurcation sets associated with the cusp catastrophe. A number of conclusions can be drawn from this diagram, the most important being the fact that instability is not limited to large uniform networks with uniform traffic. In fact the angle subtended about the origin, by the bifurcation set, is larger for the non-uniform case. This implies that instabilities occur in non-uniform networks for a larger range of traffic asymmetries than is the case for uniform capacity networks. On the other hand, the width of the bifurcation set is smaller for the non-uniform case, suggesting that a lower level of trunk reservation will be needed to stabilize them than for uniform networks. This suggests that a conservative approach to stabilizing non-uniform networks, involves using the stabilizing reservation threshold, $R^{*}$.

\section{Conclusions}

The analyses have served to confirm the hypothesis that positive feedback in the mutual overflow streams is the cause of instability. The analysis demonstrates the pervasive nature of the instability phenomenon in systems with mutual overflow. An analytic approach has been employed to ascertain the effect of a number of parameters on the network stability. The list includes, the effect of trunk group size, the number of alternate routes, routing discipline, call set-up procedure, non-uniformity of group sizes, offered load, number of hops/path, static trunk reservation levels, and peakedness in the link offered traffics. Generally we find that instability can occur for sufficiently large trunk groups and number alternate routes in mutual overflow schemes. Trunk reservation and route cancelation are powerfu] tools for stabilizing, an otherwise unstable retwork. For the asymptotic symmetric networks, a simple analytic expression has been derived for a stabilizing reservation threshold, as a function of trunk group size. It is conjectured that this provides a conservative reservation level for nonuniform networks. A curve relating a stable number of alternate routes as a function of group size has been given for the uniform network. We also conjecture that this will be a conservative rule for stabilizing nonuniform networks via route cancellation.

\section{References}

[1] Nakagome Y., Mori H., "Flexible Routing in Global Communications Network", ITC Vol. 7, pp. 426.1-426.8, 1973.

[2] Krupp R.S., "Stabilization of Alternate Routing Networks", ICC Vol. 82, pp. 31.2.1-31.2.5, 1982.

(3) Marbukh V.V., "Asymptotic Investigation of a Complete Communications Network with a Large Number of Nodes and Bypass Routes", Translated from Problemy Peredachi Informatsii, Vol. 16, No. 3, pp. 89-95, July-Sept., 1981.

[4] Mason L.G., "Symmetric Routing in Uniform Networks", INRS-Tech. Report No. 83-02, February 1983.

[5] Akinpelu J.M., "The Overload Performance of Engineered Networks with Nonhierarchical and Hierarchical Routing", ITC, Vol. 10 pp. 3.2.4.1-3.2.4.7, 8-15 June 1983.

[6] Onozato Y., Noguchi S., "On the Thrashing Cusp in Slotted Aloha Systems". IEEE Trans. on Communications. Vol. COM-33. No. 11. November 1985.

[7] Saunders P.T.. "An Introduction to Catastrophe Theory". Cambridge, University Press, 1980. 


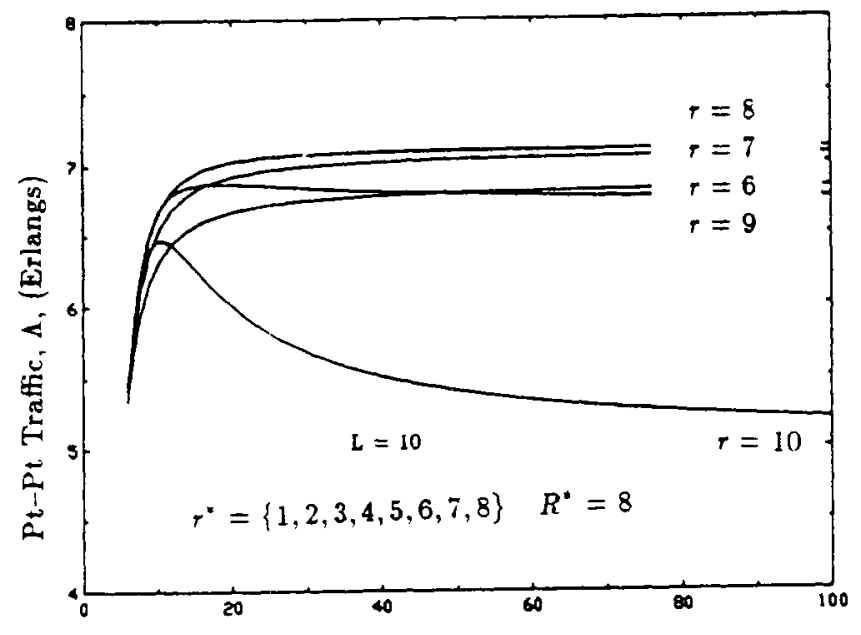

Link Offered Traffic, $Z$, (Erlangs)

Figure 1. Asymptotic Network Flows

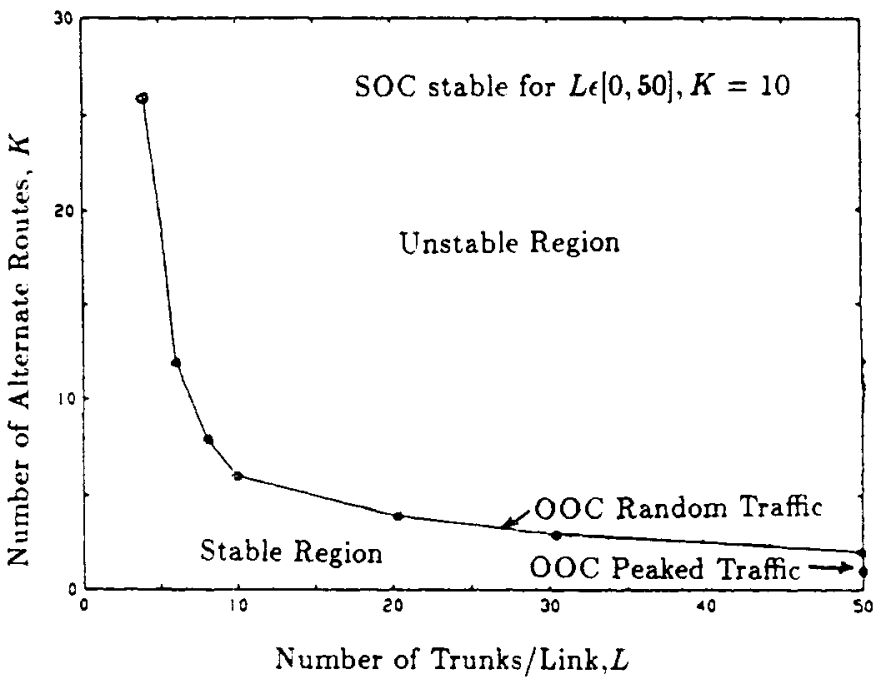

Figure 2. Iniform Finite Network Stability Boundary

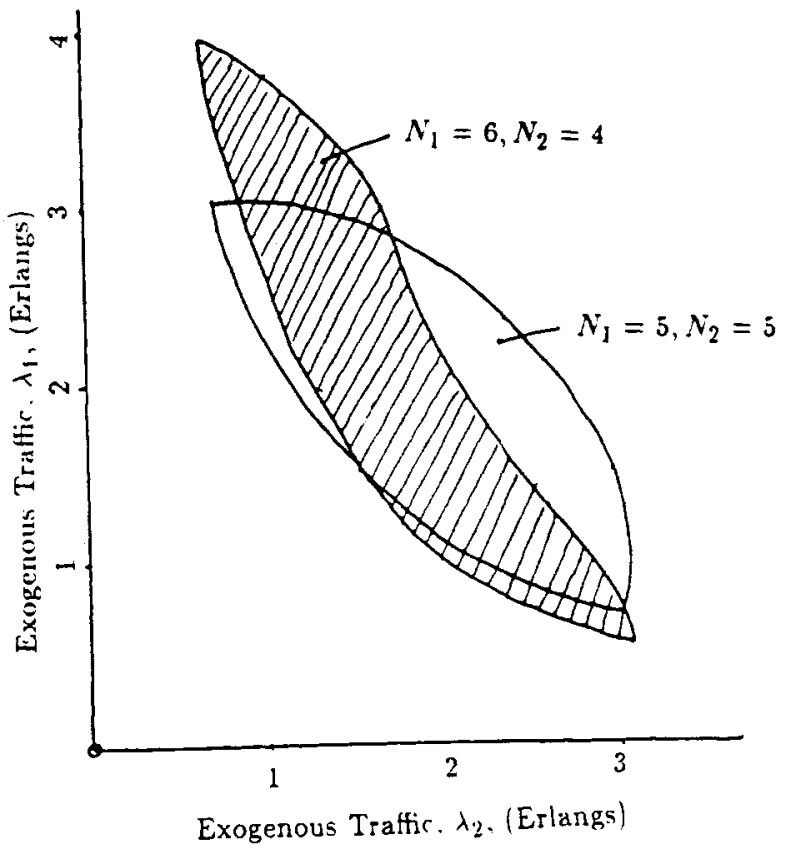

Figure 3. Non-uniform Network Bifurcation Sets 